\author{
CONVEX AND FRACTAL GEOMETRY \\ BANACH CENTER PUBLICATIONS, VOLUME 84 \\ INSTITUTE OF MATHEMATICS \\ POLISH ACADEMY OF SCIENCES \\ WARSZAWA 2009
}

\title{
FRACTAL-CLASSIC INTERPOLANTS
}

\author{
M. A. NAVASCUÉS and M. V. SEBASTIÁN \\ Department of Applied Mathematics \\ University of Zaragoza \\ C/ María de Luna 3, 50018 Zaragoza, Spain \\ E-mail:manavas@unizar.es,msebasti@unizar.es
}

\begin{abstract}
The methodology of fractal interpolation is very useful for processing experimental signals in order to extract their characteristics of complexity. We go further and prove that the Iterated Function System involved may also be used to obtain new approximants that are close to classical ones. In this work a classical function and a fractal function are combined to construct a new interpolant. The fractal function is first defined as a perturbation of a classical mapping. The additional condition of proximity to another interpolant leads to a problem of convex optimization whose solution is a fractal element with mixing properties. This procedure may be applied to the reduction of the regularity order of traditional approximants and for the computation of models with rich geometric structure.
\end{abstract}

1. Introduction. Fractal geometry provides a new insight into the approximation and modeling of natural phenomena $[1,7]$. The method of Iterated Function Systems supports the understanding and processing of complex sets [8]. Barnsley has used this methodology for the interpolation of real data [1,2]. Fractal interpolation functions (FIF's) constitute a useful tool for the approximation of experimental data. These maps possess good geometric properties providing suitable graphical representations of complex phenomena and a simple computation of the fractal dimension of their graphs. In former papers, we have proved that this method is so general that it contains other interpolation techniques as particular cases. Specifically, we have generalized some classical approximation functions such as cubic and Hermite splines by means of fractal interpolation [10, 11].

A new characteristic of this kind of approximant is the non-smoothness of the functions obtained [5]. This feature allows to mimic real-world signals showing a generally rough aspect. Another important fact is that the graph of these interpolants possesses a fractal

2000 Mathematics Subject Classification: Primary 28A80; Secondary 65D05, 58C05.

Key words and phrases: iterated function systems, affine fractal interpolation functions. The paper is in final form and no version of it will be published elsewhere. 
dimension, and this number can be used to measure the complexity of a signal, allowing an automatic comparison of data such as electroencephalographic recordings [12].

In this paper we develop a method for the computation of an interpolant combining aspects of classical and fractal functions. The fractal function is defined first as a perturbation of a classical function. The additional condition of proximity to another interpolant provides a problem of convex optimization whose solution is a fractal element with mixing properties. The procedure may be applied to the reduction of the regularity order of traditional approximants, and to the computation of models with rich geometric structure.

2. Fractal functions. In former papers ([14], [15]), we have studied an Iterated Function System $\left\{w_{n}(t, x)\right\}_{n=1}^{N}$ defined on the set $C=I \times \mathbb{R}$, where $I$ is a compact interval, $I=[a, b] \subset \mathbb{R}$. The maps $w_{n}(t, x)$ are defined by

$$
w_{n}(t, x)=\left(L_{n}(t), F_{n}(t, x)\right) \quad \forall n=1,2, \ldots, N
$$

where

$$
\left\{\begin{array}{l}
L_{n}(t)=a_{n} t+b_{n}, \\
F_{n}(t, x)=\alpha_{n} x+q_{n}(t) .
\end{array}\right.
$$

The system is associated with a partition of the interval $I$

$$
\Delta: a=t_{0}<t_{1}<\cdots<t_{N}=b .
$$

The coefficients $a_{n}, b_{n}$ are defined in terms of the nodes of the partition as

$$
a_{n}=\frac{t_{n}-t_{n-1}}{t_{N}-t_{0}}, \quad b_{n}=\frac{t_{N} t_{n-1}-t_{0} t_{n}}{t_{N}-t_{0}},
$$

and $F_{n}(t, x)$ satisfies some Lipschitz conditions ([1]). The multiplier $\alpha_{n}$ is a vertical scale factor of the transformation, such that $-1<\alpha_{n}<1 . \alpha=\left(\alpha_{1}, \alpha_{2}, \ldots, \alpha_{N}\right)$ is the scale vector.

TheOREM $1([1,2])$. The iterated function system (IFS) defined above admits a unique attractor $G$. $G$ is the graph of a continuous function $h: I \rightarrow R$ interpolating the data $\left(h\left(t_{n}\right)=x_{n}\right.$, for all $\left.n=0,1, \ldots, N\right)$.

The previous function is called a fractal interpolation function (FIF) corresponding to $\left\{\left(L_{n}(t), F_{n}(t, x)\right)\right\}_{n=1}^{N}$. It satisfies the functional equation:

$$
h(t)=F_{n}\left(L_{n}^{-1}(t), h \circ L_{n}^{-1}(t)\right) .
$$

In this paper we study a particular case of a Fractal Interpolation Function (FIF). The map $q_{n}$ is defined as

$$
q_{n}(t)=g \circ L_{n}(t)-\alpha_{n} b(t)
$$

where $g$ and $b$ are continuous functions, $g, b: I \rightarrow \mathbb{R}$, such that $b\left(t_{0}\right)=g\left(t_{0}\right), b\left(t_{N}\right)=$ $g\left(t_{N}\right)$.

The attractor of the system is the graph of a continuous function $g^{\alpha}: I \rightarrow \mathbb{R}$ which interpolates to $g$ at the nodes of the partition,

$$
g^{\alpha}\left(t_{n}\right)=g\left(t_{n}\right) \quad \forall n=0,1, \ldots, N .
$$




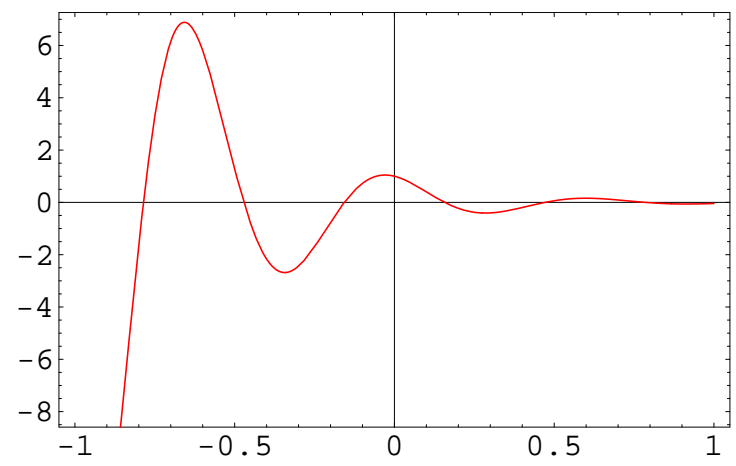

Fig. 1. Original function $g$

(See Figures 1, 2). The mapping $g^{\alpha}$ satisfies the functional equation (3)

$$
g^{\alpha}(t)=g(t)+\alpha_{n}\left(g^{\alpha}-b\right) \circ L_{n}^{-1}(t) \quad \forall t \in I_{n}
$$

Let $\mathcal{G}$ be the set of continuous functions

$$
\mathcal{G}=\left\{f \in \mathcal{C}[a, b]: f\left(t_{0}\right)=g\left(t_{0}\right), f\left(t_{N}\right)=g\left(t_{N}\right)\right\}
$$

$\mathcal{G}$ equipped with the uniform norm is a complete metric space. Define a mapping $T^{\alpha}$ : $\mathcal{G} \rightarrow \mathcal{G}$ by:

$$
\left(T^{\alpha} f\right)(t)=F_{n}\left(L_{n}^{-1}(t), f \circ L_{n}^{-1}(t)\right)
$$

for all $t \in\left[t_{n-1}, t_{n}\right], \quad n=1,2, \ldots, N$. It is a contraction mapping on the metric space $\left(\mathcal{G},\|\cdot\|_{\infty}\right)$ and possesses a unique fixed point on $\mathcal{G}$, that is the FIF $g^{\alpha}$.

The uniform distance between $g^{\alpha}$ and $g$ is bounded in terms of the scale vector $([12])$ and the map $b$,

$$
\left\|g^{\alpha}-g\right\|_{\infty} \leq \frac{|\alpha|_{\infty}}{1-|\alpha|_{\infty}}\|g-b\|_{\infty}
$$

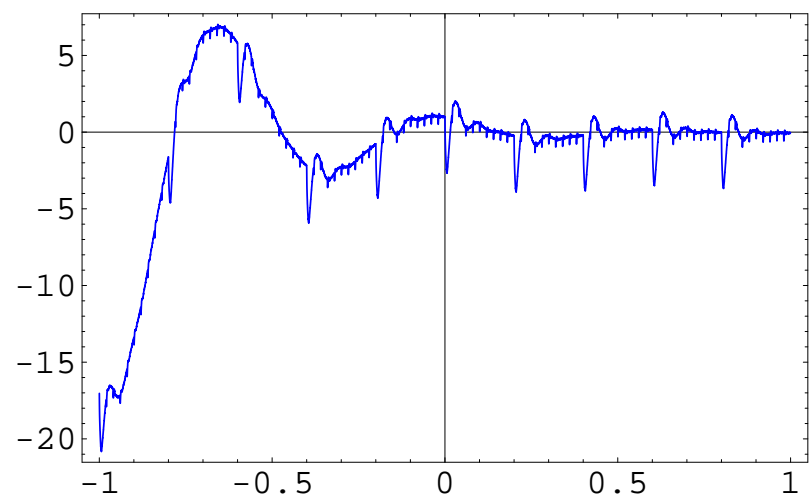

Fig. 2. Fractal function associated to $g$ with respect to $\alpha=(0.15,0.15, \ldots)$ and $b(t)$ a line 
where $\|\cdot\|_{\infty}$ is the uniform norm defined as

$$
\|f\|_{\infty}=\max \{|f(t)|: t \in I\}
$$

and

$$
|\alpha|_{\infty}=\max \left\{\left|\alpha_{n}\right|: n=1,2, \ldots, N\right\} .
$$

The scale vector gives a degree of freedom to the function $g$, allowing us to modify its properties or to approach a given function.

3. Construction of hybrid fractal interpolants. In this section we develop a method for the construction of a new interpolant as described above. We follow arguments similar to those of [9], for a different IFS.

Theorem 2 (Collage Theorem [2]). Let $(X, d)$ be a complete metric space and let $T$ be a contraction map with contraction factor $c \in[0,1)$. Then, for any $f \in X$

$$
d(f, \tilde{f}) \leq \frac{1}{1-c} d(f, T f)
$$

where $\tilde{f}$ is the fixed point of $T$.

We consider two classical interpolants $(S$ and $P$ ) of a set of data. We construct the fractal function $S^{\alpha}$ associated to $S$, defined in the previous section. Now we apply the collage theorem for $f=P, \tilde{f}=S^{\alpha}$ and $T=T^{\alpha}$.

The distance here is the uniform metric and $T=T^{\alpha}$ is the contraction (7), so that $\left\|T^{\alpha} P-P\right\|_{\infty}<\varepsilon$ implies $\left\|P-S^{\alpha}\right\|_{\infty}<\frac{\varepsilon}{1-|\alpha|_{\infty}}$ and $S^{\alpha}$ will be a fractal interpolant close to $P$.

We may set the problem of finding $\alpha^{*}$ such that

$$
\alpha^{*}=\min _{\alpha}\left\|T^{\alpha} P-P\right\|_{\infty}=\min _{\alpha} c(\alpha)
$$

where $|\alpha|_{\infty} \leq \delta<1$.

The classical interpolants (polynomial, spline) are piecewise smooth and consequently by the definition of $T^{\alpha}$, so is $T^{\alpha} f-f$. The function $c(\alpha)$ is non-differentiable in general, but its convexity can be proved and thus, the problem

$$
(C P)\left\{\begin{array}{l}
\min _{\alpha} c(\alpha) \\
|\alpha|_{\infty} \leq \delta<1
\end{array}\right.
$$

is a constrained convex optimization problem. The existence of solution is clear if $c$ is a continuous function as $\mathcal{B}_{\delta}=\left\{\alpha \in \mathbb{R}^{N} ;|\alpha|_{\infty} \leq \delta<1\right\}$ is a compact set of $\mathbb{R}^{N}$. Let us see that $c$ is continuous, and $(C P)$ convex.

The operator $T^{\alpha}$ takes here the form

$$
T^{\alpha} f(t)=S(t)+\alpha_{n}(f-b) \circ L_{n}^{-1}(t)
$$

for all $t \in I_{n}$ and $f \in \mathcal{G}$.

Proposition 1. Let $P \in \mathcal{G}$ be given and $\mathcal{B}_{\delta}=\left\{\alpha \in \mathbb{R}^{N} ;|\alpha|_{\infty} \leq \delta<1\right\}$. The map $g: \mathcal{B}_{\delta} \rightarrow \mathcal{G}$ defined by $g(\alpha)=T^{\alpha} P$ such that

$$
T^{\alpha} P(t)=S(t)+\alpha_{n}(P-b) \circ L_{n}^{-1}(t)
$$

for all $t \in I_{n}$, is continuous with respect to $\alpha$. 
Proof. According to (12) if $\alpha, \beta \in \mathcal{B}_{\delta}$ and for $t \in I_{n}$,

$$
\begin{aligned}
& T^{\alpha} P(t)=S(t)+\alpha_{n}(P-b) \circ L_{n}^{-1}(t), \\
& T^{\beta} P(t)=S(t)+\beta_{n}(P-b) \circ L_{n}^{-1}(t),
\end{aligned}
$$

then

$$
\left|T^{\alpha} P(t)-T^{\beta} P(t)\right| \leq\left|\alpha_{n}-\beta_{n}\right|\|P-b\|_{\infty}
$$

and

$$
\left\|T^{\alpha} P-T^{\beta} P\right\|_{\infty} \leq|\alpha-\beta|_{\infty}\|P-b\|_{\infty},
$$

SO

$$
\|g(\alpha)-g(\beta)\|_{\infty} \leq|\alpha-\beta|_{\infty}\|P-b\|_{\infty},
$$

hence $g(\alpha)$ is Lipschitz with constant $M=\|P-b\|_{\infty}$ and the continuity of $g$ is deduced.

Consequence 1. $c(\alpha)=\left\|T^{\alpha} P-P\right\|_{\infty}=\|g(\alpha)-P\|_{\infty}$ is continuous because it is obtained by adding and composing continuous functions.

CONSEQUence 2. The problem $(C P)$ admits at least one solution.

Proposition 2. The function

$$
c(\alpha)=\left\|T^{\alpha} P-P\right\|_{\infty}
$$

is convex.

Proof. Let $\lambda \in \mathbb{R}$ be such that $0 \leq \lambda \leq 1$, and $\alpha^{1}, \alpha^{2}$ scale vectors. Since any constant $a$ can be expressed as $a=\lambda a+(1-\lambda) a$, and by the equality (12),

$$
\begin{aligned}
& c\left(\lambda \alpha^{1}+(1-\lambda) \alpha^{2}\right) \\
= & \max \left\{\left|T^{\lambda \alpha^{1}+(1-\lambda) \alpha^{2}} P(t)-P(t)\right| ; t \in I\right\} \\
= & \max _{1 \leq n \leq N}\left\{\left|S(t)-P(t)+\left(\lambda \alpha_{n}^{1}+(1-\lambda) \alpha_{n}^{2}\right)(P-b) \circ L_{n}^{-1}(t)\right| ; t \in I_{n}\right\} \\
\leq & \max _{1 \leq n \leq N}\left\{\lambda\left|S(t)-P(t)+\alpha_{n}^{1}(P-b) \circ L_{n}^{-1}(t)\right|\right. \\
& \left.+(1-\lambda)\left|S(t)-P(t)+\alpha_{n}^{2}(P-b) \circ L_{n}^{-1}(t)\right| ; t \in I_{n}\right\} \\
\leq & \lambda\left\|T^{\alpha^{1}} P-P\right\|_{\infty}+(1-\lambda)\left\|T^{\alpha^{2}} P-P\right\|_{\infty}=\lambda c\left(\alpha^{1}\right)+(1-\lambda) c\left(\alpha^{2}\right) .
\end{aligned}
$$

Proposition 3. The set $\mathcal{B}_{\delta}=\left\{\alpha \in \mathbb{R}^{N} ;|\alpha|_{\infty} \leq \delta\right\}$ is convex.

As a result of the former propositions, $(C P)$ is a problem of constrained convex optimization with some solution.

If $\alpha^{*}$ is the optimum scale, the expression $c\left(\alpha^{*}\right) /\left(1-\left|\alpha^{*}\right|_{\infty}\right)$ provides an upper bound for the uniform distance $\left\|S^{\alpha^{*}}-P\right\|_{\infty}$ according to the Collage Theorem. Here $P$ is a classical interpolant and $S^{\alpha^{*}}$ is the fractal function perturbed from $S$ and close to $P$.

THEOREM 3. If $X(t)$ is the original continuous function providing the interpolation data and $\alpha^{*}$ is the optimum scale, the following error estimate is obtained:

$$
\left\|X-S^{\alpha^{*}}\right\|_{\infty} \leq E_{P}+\frac{c\left(\alpha^{*}\right)}{1-\left|\alpha^{*}\right|_{\infty}}
$$

where $E_{P}$ is an upper bound of the interpolation error corresponding to $P$. 


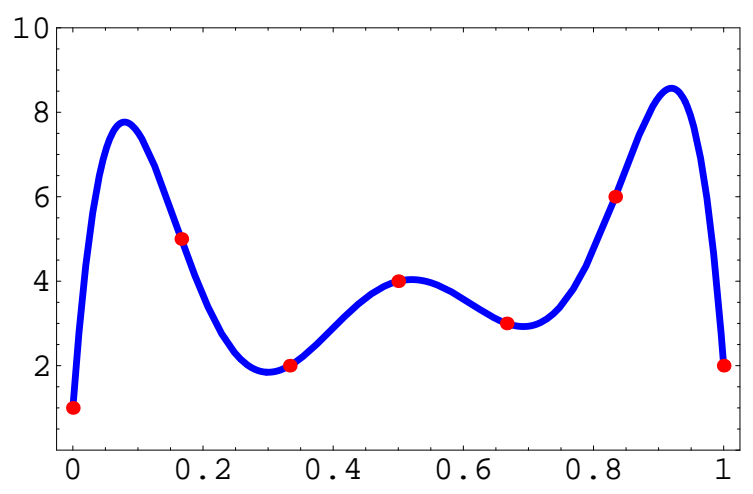

Fig. 3. Polynomial interpolant $P$

Proof.

$$
\left\|X-S^{\alpha^{*}}\right\|_{\infty} \leq\|X-P\|_{\infty}+\left\|P-S^{\alpha^{*}}\right\|_{\infty}
$$

from which the result is deduced.

Proposition 4. If, in the problem $(C P)$, we set $\delta=\delta(h)$, tending to zero with $h$, and $S$ and $P$ are convergent interpolants, then so is $S^{\alpha^{*}}$.

Proof. If $\delta \rightarrow 0$, then

$$
c\left(\alpha^{*}\right) \rightarrow c(0)=\|S-P\|_{\infty}
$$

according to (12). If $P$ and $S$ are convergent, both terms in (15) tend to zero and $S^{\alpha^{*}}$ is convergent.

THEOREM 4. If $X(t)$ is the original continuous function providing the data and $\alpha^{*}$ is the optimum scale, the following error estimate is obtained:

$$
\left\|X-S^{\alpha^{*}}\right\|_{\infty} \leq E_{S}+\frac{\left|\alpha^{*}\right|_{\infty}}{1-\left|\alpha^{*}\right|_{\infty}}\|S-b\|_{\infty}
$$

where $E_{S}$ is an upper bound of the error corresponding to $S$.

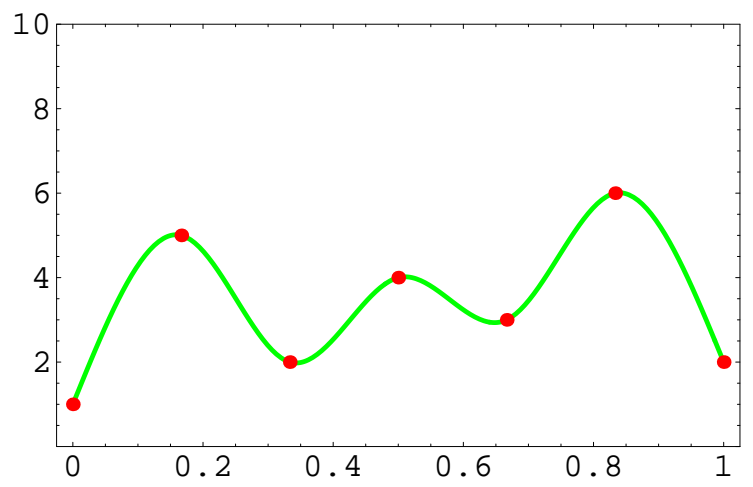

Fig. 4. Natural cubic spline $S$ 


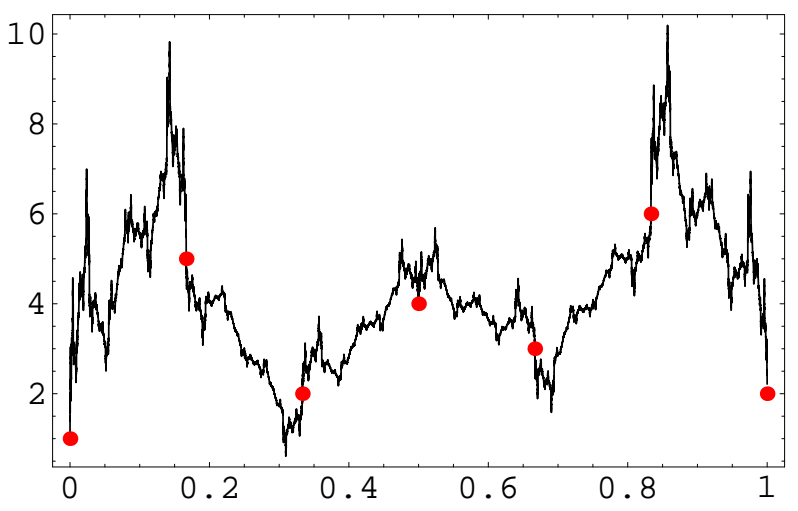

Fig. 5. Fractal cubic spline $S^{\alpha^{*}}$ from $P$ and $S$

Proof.

$$
\left\|X-S^{\alpha^{*}}\right\|_{\infty} \leq\|X-S\|_{\infty}+\left\|S-S^{\alpha^{*}}\right\|_{\infty}
$$

Applying the inequality (8) for $g=S$ and $\alpha=\alpha^{*}$ the result is proved.

Consequence 3. Choosing $\delta=\delta(h) \rightarrow 0$ and a convergent interpolant $S$, both terms of (16) tend to zero as does $S^{\alpha^{*}}$. Note that only one convergent interpolant is needed; and this result improves the previous Proposition.

\section{Some comments:}

- The method transforms a (possibly) non-convergent interpolant $P$ into a convergent $S^{\alpha^{*}}$. Particular case: If the original function $X$ is smooth enough, its (natural) cubic spline $S$ converges and thus $S^{\alpha^{*}}$ does also.

- The diversity of options $\left(P, S, S^{\alpha^{*}}\right)$ allows us to choose the best one for an approximation-optimization problem.

- The methodology described may be used to reduce the order of regularity of classical interpolants.

- Choosing $b$ suitably, $S^{\alpha^{*}}$ may be differentiable ([13]). In this case, the approximation of the derivatives of $X$ is also possible.

- The method has new geometric possibilities: In the non-smooth case, the graph of $S^{\alpha^{*}}$ has a non-integral fractal dimension and this parameter provides an index for experimental signals (See Figures 3, 4, 5).

\section{References}

[1] M. F. Barnsley, Fractal functions and interpolation, Constr. Approx. 2 (1986), 303-329.

[2] M. F. Barnsley, Fractals Everywhere, Academic Press, 1988.

[3] M. F. Barnsley and D. Saupe, Fractals in Multimedia, I.M.A. 132, E. R. Vrscay (eds.), Springer, 2002.

[4] M. F. Barnsley, Superfractals, Cambridge University Press, 2006. 
[5] S. Chen, The non-differentiability of a class of fractal interpolation functions, Acta Math. Sci. 19 (1999), 425-430.

[6] E. W. Cheney, Approximation Theory, AMS Chelsea Publ., 1966.

[7] K. J. Falconer, Fractal Geometry, Mathematical Foundations and Applications, Wiley, 1990.

[8] J. E. Hutchinson, Fractals and self similarity, Indiana Univ. Math. J. 30 (1981), 713-747.

[9] D. La Torre and M. Rocca, Approximating continuous functions by iterated functions systems and optimization, Int. Math. J. 2 (2002), 801-811.

[10] M. A. Navascués and M. V. Sebastián, Some results of convergence of cubic spline fractal interpolation functions, Fractals 11 (2003), 1-7.

[11] M. A. Navascués and M. V. Sebastián, Generalization of Hermite functions by fractal interpolation, J. Approx. Th. 131 (2004), 19-29.

[12] M. A. Navascués and M. V. Sebastián, Fitting curves by fractal interpolation: an application to the quantification of cognitive brain processes, in: Thinking in Patterns: Fractals and Related Phenomena in Nature, M. M. Novak (ed.), World Sci., 2004, 143-154.

[13] M. A. Navascués and M. V. Sebastián, Smooth fractal interpolation, Journal of Inequalities and Applications 2006, Art. ID 78734, 20 pp.

[14] M. A. Navascués, Fractal polynomial interpolation, Z. Anal. Anwendungen 24 (2005), 401-418.

[15] M. A. Navascués, Fractal trigonometric approximation, Electron. Trans. Numer. Anal. 20 (2005), 64-74. 